\title{
Special issue with selected papers from 2019 Brazilian Symposium on Computer Engineering (SBESC 2019)
}

\author{
Ivan Muller ${ }^{1} \cdot$ Marcelo Götz ${ }^{2}$ \\ Published online: 29 May 2021 \\ (C) The Author(s), under exclusive licence to Springer Science+Business Media, LLC, part of Springer Nature 2021
}

The Embedded Systems track of the IX Brazilian Symposium on Computing Systems Engineering (SBESC), which took place in Natal, Rio Grande do Norte, Brazil, received a considerable number of high-quality papers. Considering the rank produced by the Program Committee, we invited the best ranked papers to submit extended versions to his special issue. After a strict review process, we selected three articles for inclusion in this special issue.

The first article is entitled "An Analysis of the Gateway Integrity Checking Protocol from the perspective of Intrusion Detection Systems" by Mateus Martínez de Lucena, Roberto Milton Scheffel, and Antônio Augusto Fröhlich. The authors presented an original approach to analyzing the integrity of data sent by a gateway to the cloud from an Internet of Things (IoT) network. The approach is based on a gossip protocol that performs challenges that need to be answered by a subset of IoT devices. Based on the answer, the protocol can detect if the gateway is trustable or has been victimized by malicious attackers. Simulations results are provided, which analyses the time and energy overhead and the impact on the lifetime of the network when using the proposed protocol.

The second article is entitled "Synthetic Image Generation for Training Deep Learningbased Automated License Plate Recognition Systems on the Brazilian Mercosur Standard" by Gilles Silvano, Vinícius Ribeiro, Vitor Greati, Aguinaldo Bezerra, Ivanovitch Silva, Patricia Takako Endo, and Theo Lynn. The authors present a novel embedded methodology for generating large volumes of qualified Mercosur license plate images for appropriate training of a License Plate Detection process. This methodology considerably improves Deep-Learning based automated license plate detection. Practical applications on a real parking lot and traffic camera images validated the approach with sound results.

The third article is entitled "Dynamic Concurrency Throttling on NUMA Systems and Data Migration Impacts" by Janaína Schawarzrock, Michael Guilherme Jordan, Guilherme Korol, Charles C. de Oliveira, Arthur F. Lorenzon, Mateus Beck Ritzig, and Antônio Carlos S. Beck. In parallel applications a runtime strategy can be used to adjust the number of threads, depending on the parallel region of the application, aiming for the best outcome in

Ivan Muller

ivan.muller@ufrgs.br

1 Electrical Engineering Department, Universidade Federal do Rio Grande do Sul (UFRGS), Porto Alegre, RS, Brazil

2 Automation and Energy Department, Universidade Federal do Rio Grande do Sul (UFRGS), Porto Alegre, RS, Brazil 
performance or energy consumption. This strategy is called Dynamic Concurrency Throttling (DCT). This work shows that, in a Non-Uniformed Memory Access (NUMA) where data migration between nodes is necessary, it directly affects the outcome of DCT's Strategy with implications on the overall performance and energy of the application.

We would like to express our gratitude to the Technical Program Committee of SBESC for their hard work on reviewing the papers for the conference, the additional reviewers who helped us with the review process for this special issue, as well as the authors themselves for their excellent contributions.

Publisher's Note Springer Nature remains neutral with regard to jurisdictional claims in published maps and institutional affiliations. 